\title{
A Dynamic and Combined Framework for Predicting Phishing Attack
}

\author{
Antony Vijay J, Sumit Saurabh, Rajat Sharma, Sachin Roy, Sourav Roy
}

\begin{abstract}
Right now internet where every single of us is reliant or leaning upon the web/internet some or the many ways. Practically all the exercises which incorporates web based booking, paying bills, entertainment requires web and there is a ton of chances that these exercises which we perform may be utilized by programmers to get to our classified information which may offer access to our own private data. At the point when we are utilising web for a more extended span of time, then that point at which we may be trapped in phishing assault which are generally performed by expert programmers. Right now make counterfeit site which on login will divert you to their site which will store certifications to utilize them. In spite of the fact that we are as of now educated by the Cyber wrongdoing network about the phishing, there are numerous strategies, programming and systems which causes the online client to get nitty-gritty data about the assault before the assault even happens. The achievements pace of these are not exceptionally high but rather still the client can get a harsh thought regarding it. This project will expand the achievement pace of phishing location so the individuals utilizing the web are more protected and can safely utilize the web.
\end{abstract}

Keywords: Phishing, Neural Networks, Classification, Learning, Web Security.

\section{INTRODUCTION}

Right now the programmers are utilizing comparable glancing site to cause the web client to befuddle by which the client utilizes an inappropriate site to enter their classified data or certifications accordingly the programmer will have the way in to the clients data. We are utilizing Fuzzy Rough Set (FRS) instrument which utilizes three significant highlights for breaking down the information. Presently this information will be embedded into three distinctive phishing recognition components. The first is a multi-layer perceptron, second is Random backwoods tree calculation and the last one is successive negligible optimization (SMO).

Revised Manuscript Received on April, 042020.

* Correspondence Author

J.Antony Vijay*, Assistant professor of Information technology in SRM IST, Ramapuram Campus, Chennai, Tamil Nadu, India. Email: antonyvj@srmist.edu.in

Sumit Saurabh, Department of Information Technology, SRM IST,Chennai,Tamil Nadu, India . Email: sumit1234saurabh@gmail.com

Rajat Sharma, Department of Information Technology, SRM IST,Chennai,Tamil Nadu, India. Email: Rajat4322@gmail.com

Sourav Roy, Department of Information Technology, SRM IST,Chennai,Tamil Nadu, India .Email: rathsourav4@gmail.com

Sachin Roy, Department of Information Technology, SRM IST,Chennai,Tamil Nadu, India . Email: sachinroyfunny@gmail.com

(C) The Authors. Published by Blue Eyes Intelligence Engineering and Sciences Publication (BEIESP). This is an open access article under the CC BY-NC-ND license (http://creativecommons.org/licenses/by-nc-nd/4.0/)
These classifiers will autonomously prepare the informational collection to check the proficiency of FRS for phishing identification. This preparation sets are made by extraction of various sites utilizing internet. This will lessen the measure of bogus phishing recognition, the fundamental objective is to elicit the space name from the hood wink url and afterward to compare the page rank of the area name with unique space now in the event that the rank is extraordinary, at that point the area name will consider as phishing. This strategy is extremely valuable for abstaining from phishing assault.

\section{RELATED WORK}

Generally a programmer (hacker) sends a mail to the client who is associated with the web realizing that the client is utilizing a framework which substance his/hers secret data. The client opens the mail which contains a connect to open, in the event that the client opens the connection, at that point it will divert the client to a site page that has similar informational indexes as the first site has to cause the client to confound about the current unique website. After this the phishing assault will occur by which the subtleties entered by the client will presently be offered back to the programmer (hacker). This data may be utilized in an incorrect manner to trick the client. In the technique for existing framework we are utilizing the real information cautiously and the most fundamental part is to gather the crude informational collection utilizing the component determination and machine learning. Feature extraction is a procedure of separating highlights and other significant angles that a site has and afterward it makes a comparable substance on another site.

\section{A. GA Population:}

Right now picking features are one of the significant jobs, for this base application is video. To get lip movement data we need three-dimensional progress. Lip-perusing is completely founded on getting the edge highlight and standardization, however there are consistently plausibility that we may lose the Data during institutionalization. For legal investigation hereditary calculation input is being utilized. For compacting highlight size is utilized for diminishing preparing and testing time. CUAVE and TULIPS database are being utilized for digit vocalization. WEKA programming is utilized to contrast and the outcome.

\section{B. Methods and Implementation:}

There is one instrument and classifiers to order the informational indexes. Likewise, different example informational indexes are utilized to make a web based putting away framework.

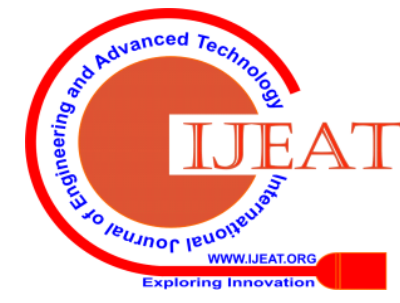




\section{A Dynamic and Combined Framework for Predicting Phishing Attack}

An apparatus named Fuzzy Rough Set is utilized alongside three classifiers named Multi-layer perceptron, Random forest, Sequential Minimal Optimization.

\section{- Fuzzy Rough Set:}

In the suggested framework all the three informational indexes are utilized to pick the successful properties and uses it to the FRS (Fuzzy Rough Set). The picked properties are gathered for phishing identification. The new framework check the capacity of the FRS properties decision by embedding's test informational collection to every one of the methods (SMO, Multilayer perceptron, Random Forest). An internet putting away framework is made by the example informational collection which are utilized to pull-out site properties. The past work esteems are utilized to set the figure of the hyper parameter.

The new framework expands the expectation achievement pace of the phishing detectors. The fundamental objective is to pull-out the space name with page rank from the focused on site and do a correlation check with the first area page rank. If the contrast between the genuine area name and the authentic area name is extremely high, at that point it will be set apart as phishing site.

\section{- Multilayer Perceptron:}

A multilayer perceptron (MLP) is a group of calculated fake regressor where a not-decided layer (non-visible layer) is taken care of that likewise has a sigmoid function. Multiple shrouded layers can be utilized to make the design deep. It has a sandwich structure it has an information layer and a yield layer and between these two it has various concealed layer. From the outset a little information is embedded into the information layer then the yield layer does some forecast about the information and concealed layers are utilized to make design profound. The issues in machine learning (supervised learning) are likewise illuminated by multi-layer perceptron. Their preparing has been done as a couple or a lot of info yield and get the information on connection between the informational collections of ins and out. These guidelines are for tuning of parameters, or burdens and lengths to expand the achievement rate. Root mean Squared error (RMSE) is one of the manner in which is utilized to recognize and amend the missteps in the yield.

\section{- Random Forests:}

Random forest implies irregular choice which is made by this calculation like taking irregular choice on premise of meeting some specific circumstances. The choice it takes changes dependent on the conditions. It has an extremely high precision and it can likewise deal with enormous properties with little examples. It makes relapse trees dependent on the blending of Bagging and irregular determination calculations. A forest is an only an assortment of trees and greatest no. Of trees implies all the more profound woodland. In like manner, Random forest is only an assortment of dynamic trees and greatest no. Of trees implies progressively exact choice about the circumstance. It first finds all the arrangements and afterward picks the most exact choice. This strategy is definitely more superior to anything single tree as numerous trees take out the over-fitting by contrasting all the outcomes.

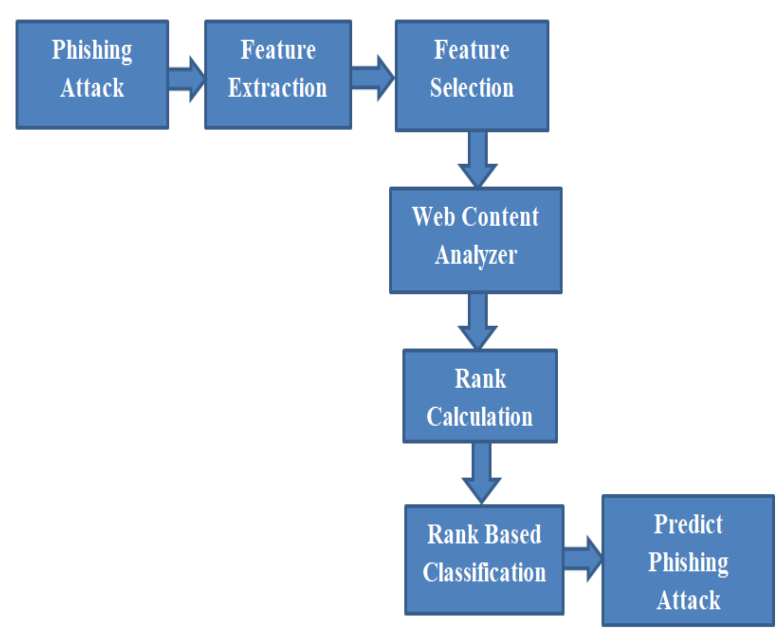

Fig. 1.Basic Architecture

\section{MODULES}

\section{A. Data Assessment:}

Right now, the factors are found individually. There are few sorts of variable to execute uni-variable examination, unmitigated and consistent. Various are the outcome of the straight out and consistent variable: The connection between two factors are bring out by Bi-variate investigation. Right now, for joint effort and non-cooperation among the factors at an extremely local level. Bi-variate examination can be executed for any blending of absolute and ceaseless factors. The blending can be: Categorical and Categorical, Categorical and Continuous and Continuous and Continuous. In the examination procedure, different systems can be utilized to separate these blending.

Ceaseless and Continuous: We need to discover the dissipate plot, when Bi-variable examination is done among the two Continuous variable. The connection among these two variables can be found through this sort of casual way. Dissipate plot design determine the connection among factors. This kind of relationships are for the most part of two sorts, Linear and Non-straight.

Wrong expectation in the after-effect of the examination is a direct result of missing information. Due to missing information, we can't set up the connection among the factors.

\section{C.Preprocessing:}

This progression has a significant effect in the last doing of the framework also. The name of the underlying advance of Natural Language Processing (NLP) is words Pre-processing.

In spite of the fact that it has an incredible effect, it doesn't get enough notification in the profound learning. The effect of straightforward content preprocessing is recognized right now. A far-reaching assessment on standard criteria is done dependent on the content arrangement and affectability investigation. There are a noteworthy variable pre-processing methods among the token. The first and the objective website page is analysed dependent on the substance similitude.

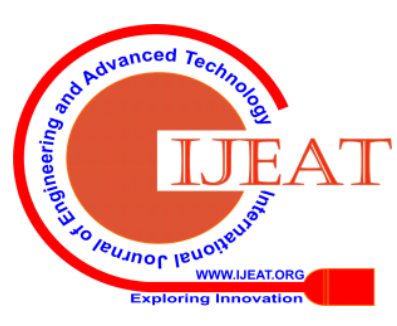


Here, the Term Frequency/Inverse archive Frequency is utilized. The terms are contrasted of the first site and the objective site by TF/IDF. Another approach to do the correlation is to take screen capture and afterward do the further procedure. We need to spare the information got from the screen capture. At that point forward the information to an internet searcher to get the objective page's position. After that the truth of the site's substance can be contrasted and the first site. Google picture database can be utilized to amend the site logo. Likewise, the rundown of spam sites is accessible in the Google database and it is refreshed habitually. Be that as it may, issue is that an as of late made phony site url won't be right now. This unlisted as of late made site escape unidentified with this methodology. Google additionally obstructs the spam sites and the client can not open it. The page rank calculation utilizes the Google's Page-Rank esteem which anybody can jump on the web.

\section{Feature Selection:}

There are various quality in the Heuristic examination, and they named as Web content trait, Web traffic property, URL characteristic. Web crawler is utilized to complete these traits. The main indication of the image '@' and '- in target URL, since '@' in a URL is checked by the URL checker. As the real destinations doesn't utilize this image '- to an extreme and the first locales contains fewer specks separated from the phony locales utilizing numerous dabs. Along these lines, the absolute number of specks are additionally determined in a URL by the URL checker. It is likewise a duty regarding the URL checker to check for words with wrong spelling and imprint the words. Later it advances the stamped words with Levenshtein Distance(LD). The distinction among two strings is tallied dependent on the Levenshtein Distance. On the off chance that more specks are utilized and the separation between them is less, at that point the objective site might be a phishing site. Another work of URL checker is to recognize the IP of the URL and discover that it matches with unique site's URL IP or not. In the event that it doesn't coordinate with the first, at that point the site is a phony. Based on these conditions the Heuristics arrange the site as genuine or phony.

\section{Prediction:}

$33 \%$ of the instances of test are tossed out while settling on the preparation set for the choice trees which in the long run make the irregular woods to make the ideal expectation. The tossed out cases are named as out-of-pack information or OOB information. This OOB information is contributed to run unprejudiced test to recognize blunder in the Random Forest.

For the entirety of the instances of each tree all the information are poured in the irregular woods and each yield is registered and furthermore all the chance is checked. In the event that a few cases matches with different cases and places in a similar terminal code, at that point one is added to their vicinity. Finally the standardization of Proximities is finished by the tree-divison strategy. Vicinities are use clamor different parts of trees amendment as anomalies putting, missing information substitution and in the creation of perspectives on information.

Tree development is somewhat mind boggling task. We need diverse bootstrap for making each tree from the genuine informational index. Just two third of the cases are utilized in creation of the Kth tree.
All the unused cases are consolidated to group the Kth tree. Finally, take I to be the most chosen case each time case n got forgot about. The OOB botch gauge is only the proportion of times I to be not equivalent to the aggregate of normal of the case $n$.

Most of the trees in random forest which got bigger, turns down the OOB cases and figure the decisions in favour of immaculate class. After that we need to do an irregular change of the estimations of $\mathrm{m}$. Next we need to pour the cases down the choice tree. Presently we need to do a subtraction of the votes given to the correct class in the irregular permuted OOB and the votes given to the typical OOB. The appropriate response is the score of variable $\mathrm{m}$.

Standardised calculation is utilized to ascertain the mix-ups, if the readings of $m$ isn't needy starting with one tree then onto the next. We have attempted this technique for figuring in a no. of test informational indexes however the appropriate response is excessively little. Along these lines, we have chosen to ascertain the errors with the old style path by partitioning the first score with its mix-ups score to produce a z-score. It is utilized for additional forecast of the phony site joined with OOB informational indexes and relegates an incentive for the crude informational collections.

\section{IMPLEMENTATION:}

The set of rules are installed in the form of extension of Google Chrome Browser. We have used Tables to display the working principles.

It is shown in the Figure 1, it includes three sections: Unified processor, Resemblance checker and the from the given sets of prey. DOM extracting unit, CSS, Visual characteristics are three major elements present in the Unified processor. The function of CSS extractor is to get the CSS guidelines instantly from the source code which is present on the web page.

\section{A. Algorithm:}

1. Consider a suspicious page $\mathrm{Q}$ on the internet which you are giving access to.

2. Now consider $\mathrm{x}$ to be the targeted web page.

3 . Let * be the mutual resemblance among the two.

4.Allow Jse() be the determined vector of the net page.

5 . Give access to Junit() be the determined vector present on the internet page.

6. Phase I: Removing and cleaning.

7. CSS text of Q should be considered

8. Evaluate the vector jes()

9. Get the details of the irrelevant data in jes()

10. Evaluate Junit()

11. Phase II: Computing the data

12. Evaluate the complex part of the $\mathrm{Q}$

13. Compute the score of $Q$ and $L$

14. Return $\mathrm{A}(\mathrm{Q}, \mathrm{L})$

15. Phase III: Take the relevant action

16. Input is $A(Q, L)$

17. If $\mathrm{S}(\mathrm{Q}, \mathrm{L})>*$ then

18. Display Attention because of similar data

19. Else

20. Show $\operatorname{SIM}(\mathrm{Q}, \mathrm{L})$

\section{Published By:}

Blue Eyes Intelligence Engineering

\& Sciences Publication

DOI: 10.35940/ijeat.D7030.049420

Journal Website: www.ijeat.org

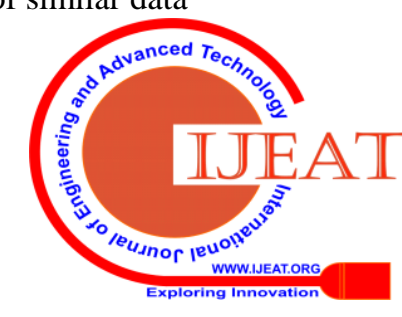




\begin{tabular}{|l|l|}
\hline \hline CPU & Intel Core i5-3210M, 2.5GHz \\
Memory & 4GB RAM \\
OS & $\begin{array}{l}\text { Windows 10-(64-bit) } \\
\text { Browser }\end{array}$ \\
& $\begin{array}{l}\text { Google Chrome 58.0.3029.110 } \\
\text { (64bit) }\end{array}$ \\
\hline
\end{tabular}

Table- 1: System Configuration

After this rest of the CSS rule will be changed to the given representation and will be transferred to the testing unit.It stores all the URL that contains the similar data from the other website as well. At this time we are actually making the use of those website the are more likely to be attacked by the phishing unit or the website that are in there target. The use of calculator is that it tells about the similarity between these website and after that the checker checks the same values in these pages. After that the result is being send to the Decision maker unit where we check the final output.

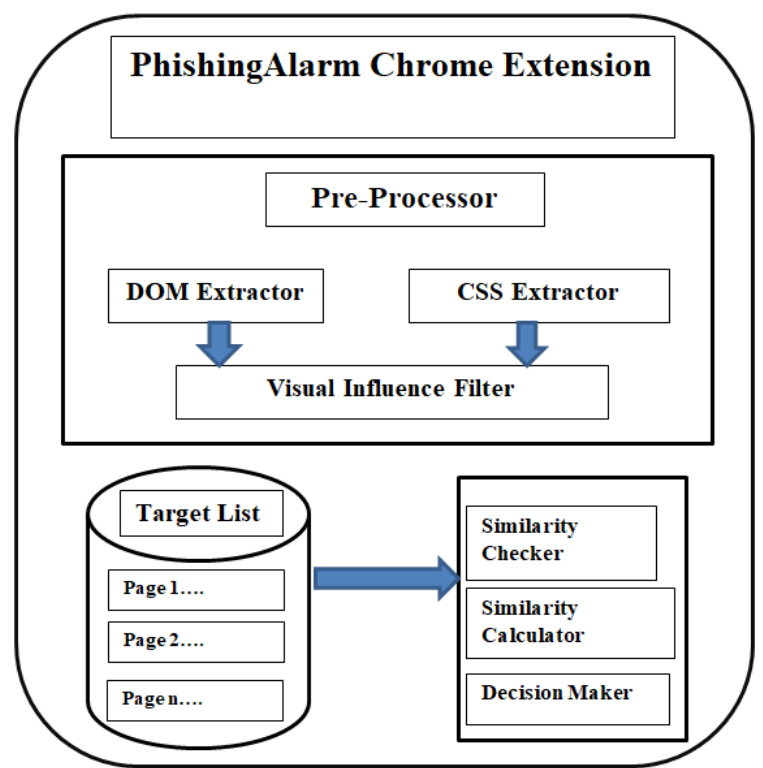

Fig. 2.Full Structure of Fake Page Detection Alarm

\begin{tabular}{|c|c|c|c|c|}
\hline \multicolumn{2}{|c|}{ Target list } & \multicolumn{3}{|c|}{$\begin{array}{l}246 \text { whitelisted } \\
\text { webpages }\end{array}$} \\
\hline \multicolumn{2}{|c|}{ Sample resource } & \multicolumn{3}{|c|}{ phistank.com } \\
\hline & & \multicolumn{2}{|c|}{$\begin{array}{l}\text { Threshold } \\
\text { selecting }\end{array}$} & Evaluation \\
\hline \multirow{3}{*}{$\begin{array}{l}\text { Phishing } \\
\text { sample }\end{array}$} & Raw & \multicolumn{2}{|c|}{6192} & 3115 \\
\hline & Valid & \multicolumn{2}{|l|}{1258} & \multirow[t]{2}{*}{289} \\
\hline & & $\begin{array}{l}\text { Set } \\
1: \\
547\end{array}$ & $\begin{array}{l}\text { Set } \\
2: \\
711\end{array}$ & \\
\hline
\end{tabular}

Table- 2: Experiment Data

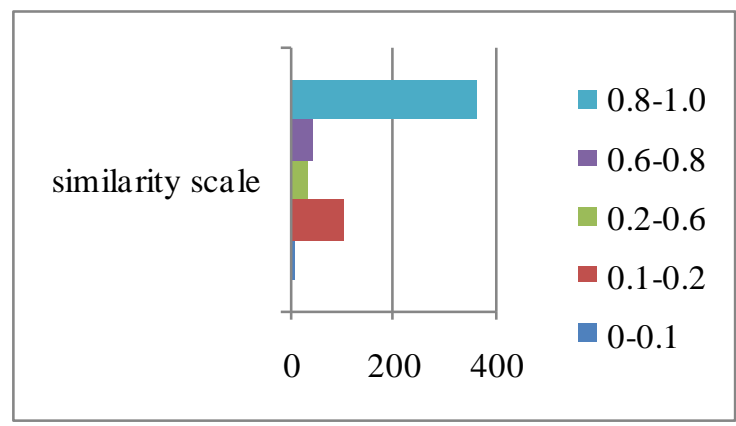

Fig.3. Resemblance reading among original pages and fake pages.

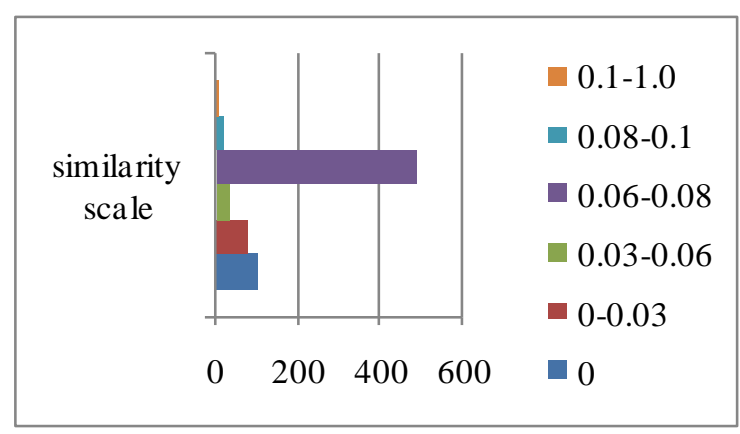

Fig.4. Resemblance reading among fake pages and their non-targets.

\section{RESULT AND DISCUSSION}

In this paper we can conclude that we are actually trying to eliminate phishing in our data. This method is one of the best to reduce this because it gives better outcome then the tools which are already existing in our own daily life. As we can see from that graph that it gives a graphical representation of the resemblance between the Original pages and fake pages. By using this we can actually estimate the amount of original data that are being copied by other websites as a result it may lead to transparency in our own data by which our credentials become unsecured. The above graph also shows resemblance among fake pages and no-target. Once the user gets an overview then he can actually protect his data because now it is already aware about these phishing attacks. This system will definitely increase the security, confidentiality and the integrity of the user by which he can feel more secure while using the Internet. One of the most important thing is to Analyse the web content every time when we are about to use a web pages, sometimes there are fake web pages which have the same view and text as the original page so as to fool the client. If necessary actions with this tool are taken, then the client will not have any threat against the phishing attack

\section{CONCLUSION}

The fundamental objective of a phishing identifier is to protect the client from different phony sites or tricks. As not ll the individual doesn't know about this quiet assault we need an all-around planned phishing apparatus to forestall the phishing assault and guard the client.

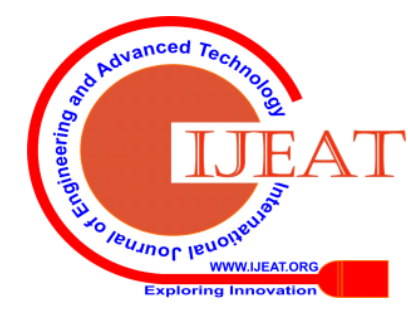


In spite of the fact that there are numerous instruments accessible like Heuristic rundown based location or AI approach they are not powerful enough. The rundown based methodology has a bogus disturbing rate at high and the AI based methodology has a decent effectiveness yet it can't recognize the new picture based phishing assault. Along these lines, we have made an ideal phishing identifier, which can distinguish counterfeit substance conveying inserted objects. Our framework will be made so that it will have a decent similarity among time-unpredictability and distinguishing productivity.

\section{REFERENCES}

1. N. Shrestha, R. K. Kharel, J. Britt and R. Hasan, "High- performance 3Classification of Phishing URLs Using a Multi-modal Approach with MapReduce”, 2015 IEEE World Congress on Services, New York, NY, 2015, pp. 206-212. [6] E. H. Chang, K. L. Chiew, S. N. Sze and W. K. Tiong, "Phishing Detection via Identication of Website Identity", 2013 International Conference on IT Convergence and Security (ICITCS), Macao, 2013, pp. 1-4. W.-K. Chen, Linear Networks and Systems (Book style). Belmont, CA: Wadsworth, 1993, pp. 123-135.

2. M. Aydin and N. Baykal, "Feature Extraction and Classification Phishing Websites Based on URL" , 2015 IEEE Conference on Communications and Network Security (CNS), Florence, 2015, pp. 769-770. B. Smith, "An approach to graphs of linear forms (Unpublished work style)," unpublished.

3. J.Antony Vijay, 'A Cost Improvement Rule Victimisation, International Journal of Recent Technology and Engineering (IJRTE), ISSN: 2277-3878, Volume-8 Issue-6, March 2020,pp 2604-2607.

4. P. Singh, N. Jain and A. Maini, "Investigating the Effect Of Feature Selection and Dimensionality Reduction On Phishing Website Classification Problem", 2015 1st International Conference on Next Generation Computing Technologies (NGCT), Dehradun, 2015, pp. 388-393. J. Wang, "Fundamentals of erbium-doped fiber amplifiers arrays (Periodical style-Submitted for publication)," IEEE J. Quantum Electron., submitted for publication.

5. M. A. U. H. Tahir, S. Asghar, A. Zafar and S. Gillani "A Hybrid Model to Detect Phishing-Sites using Supervised Learning Algorithms", 2016 International Conference on Computational Science and Computational Intelligence (CSCI), Las Vegas, NV, 2016, pp. 1126-1133. Y. Yorozu, M. Hirano, K. Oka, and Y. Tagawa, "Electron spectroscopy studies on magneto-optical media and plastic substrate interfaces(Translation Journals style)," IEEE Transl. J. Magn.Jpn., vol. 2, Aug. 1987, pp. 740-741 [Dig. $9^{\text {th }}$ Annu. Conf. Magnetics Japan, 1982, p. 301].

6. E. Buber, Demir and O. K. Sahingoz "Feature selections for the machine learning based detection of phishing websites", 2017 International Artificial Intelligence and Data Processing Symposium (IDAP), Malatya, 2017, pp. 1-5. (Basic Book/Monograph Online Sources) J. K. Author. (year, month, day). Title (edition) [Type of medium]. Volume(issue). Available: http://www.(URL)

7. Z. Dong, A. Kapadia, J. Blythe and L. J. Camp "Beyond the Lock Icon: Real-time Detection of Phishing Websites Using Public Key Certificates", 2015 APWG Symposium on Electronic Crime Research (eCrime), Barcelona, 2015, pp. 1-12. (Journal Online Sources style) K. Author. (year, month). Title. Journal [Type of medium]. Volume(issue), paging if given. Available: http://www.(URL)

8. Anti-Phishing Working Group. Accessed: Sep. 2016. [Online]. Available http://www.antiphishing.org

9. Naga Venkata Sunil and A. Sardana, "A PageRank based detection technique for phishing web sites," 2012 IEEE Symposium on Computers \& Informatics (ISCI), Penang, 2012, pp. 58-63. doi: 10.1109/ISCI.2012.6222667

10. N. Gutierrez et al., "Learning from the Ones that Got Away: Detecting New Forms of Phishing Attacks," in IEEE Transactions on Dependable and Secure Computing, vol. 15, no. 6, pp. 988-1001, 1 Nov.-Dec. 2018. doi: 10.1109/TDSC.2018.2864993

11. R. Dhamija and J.D. Tygar, "The Battle against Phishing: Dynamic SecuritySkins", Proc. Symp. Usable Privacy and Security, 2005, pp 77-88. Mobile Marketing Statistics. Accessed: Mar. 2017.

12. Sun, X., Liu, Y., Xu, M., et al.: 'Feature selection using dynamic weights for classification', Knowl.-Based Syst., 2013, 37, pp. 541-549
13. Zhao, M., Fu, C., Ji, L., et al.: 'Feature selection and parameter optimization for support vector machines: A new approach based on genetic algorithm with feature chromosomes', Expert Syst. Appl., 2011, 38, (5), pp. 5197-5204

14. Kohavi, R., John, G.H.: 'Wrappers for feature subset selection', Artif Intell., 1997, 97, (1-2), pp. 273-324

15. Das, A., Das, S.: 'Feature weighting and selection with a pareto-optimal trade-off between relevancy and redundancy', Pattern Recognit. Lett., 2017, 88, pp. 12-19

16. Varpa, K., Iltanen, K., Juhola, M.: 'Genetic algorithm based approach in attribute weighting for a medical data set', J. Comput. Med., 2014, 2014, pp. 1-11

17. Pérez-Rodríguez, J., Arroyo-Peña, A.G., García-Pedrajas, N.: 'Simultaneous instance and feature selection and weighting using evolutionary computation', Appl. Soft Comput., 2015, 37, pp. 416-443

18. Paul, S., Das, S.: 'Simultaneous feature selection and weighting - An evolutionary multi-objective optimization approach', Pattern Recogn. Lett., 2015, 65, pp. 51-59

\section{AUTHORS PROFILE}

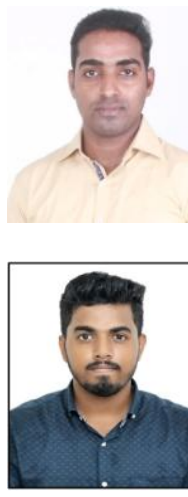

Mr. J.Antony Vijay is an Assistant Professor in Department of Information Technology, SRM IST, Ramapuram Campus, Chennai, Tamil Nadu, India and has a teaching experience of 7 years.

Sumit Saurabh is currently pursuing bachelors of technology in information technology from SRM IST, Ramapuram Campus, Chennai, Tamil Nadu, India

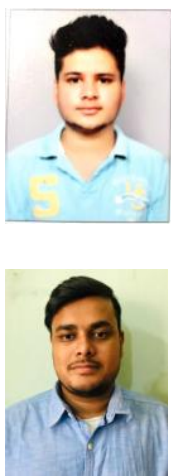

Rajat Sharma is currently pursuing bachelors of technology in information technology from SRM IST, Ramapuram Campus, Chennai, Tamil Nadu, India

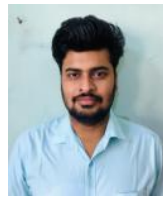

Sachin Roy is currently pursuing bachelors of technology in information technology from SRM IST, Ramapuram Campus, Chennai, Tamil Nadu, India 